\title{
Recent results from ANTARES
}

\author{
Agata Trovato $^{\mathrm{a}}$ on behalf of the ANTARES Collaboration \\ LNS-INFN, via S. Sofia 62, 95123 Catania, Italy
}

\begin{abstract}
Operating $40 \mathrm{~km}$ off the coast of France since 2007, the ANTARES detector is the largest deep-sea neutrino telescope in the Northern Hemisphere with an instrumented volume of more than 0.01 cubic kilometers. It consists of an array of 885 photomultipliers detecting the Cherenkov light induced by charged leptons produced by neutrino interactions in and around the detector. The primary goal of ANTARES is to search for astrophysical neutrinos in the TeV-PeV range. This comprises generic searches for any diffuse cosmic neutrino flux as well as more specific searches for astrophysical galactic and extragalactic sources. The search program also includes multi-messenger analyses based on time and/or space coincidences with other cosmic probes. The ANTARES observatory is sensitive to a wide-range of other phenomena, from atmospheric neutrino oscillations to dark matter annihilation.
\end{abstract}

In this contribution, recent results from the ANTARES neutrino telescope will be presented.

\section{Introduction}

After almost 50 years since the first proposal of exploiting deep-sea waters to detect cosmic high-energy neutrinos we are now at the verge of opening a neutrino era in high-energy astrophysics thanks to the results of the IceCube neutrino telescope in Antarctica that show mounting evidence of a flux of neutrinos in the $10 \mathrm{TeV}-$ $1 \mathrm{PeV}$ range exceeding the known flux of atmospheric neutrinos by a statistically significant factor [1]. The origin of this signal is however not yet clear, due to the scarceness of the detected neutrinos and mostly to the limited angular resolution of the IceCube detector. A confirmation, possibly accompanied by the identification of the sources is therefore necessary. In this context, the role of a neutrino telescope in the Northern hemisphere looking to a different part of the sky could be crucial and would help in better understanding the emission origin. Up to now the ANTARES detector is the largest deepsea neutrino telescope in the Northern Hemisphere. It will keep producing excellent results until the next generation Mediterranean Neutrino Telescope, KM3NeT, takes over.

Candidate neutrino sources in the cosmos are numerous, such as supernova remnants (SNR), Pulsar Wind Nebulae (PWN) and microquasars in the Galaxy, while possible extragalactic sources include Active Galactic Nuclei (AGN) and Gamma-Ray Bursts (GRB). Neutrinos are an optimal probe to observe high energy astrophysical phenomena, since they interact only weakly with matter and are not subject to the influence of magnetic fields. They can therefore travel long distances without being deflected or absorbed and can provide unique information on the most violent and highest energy processes in our Galaxy and far beyond. Their measurement will allow for new insights into the acceleration mechanisms, shedding light on the

${ }^{a}$ e-mail: atrovato@lns.infn.it mechanisms that are at the origin of the highest energy cosmic rays.

Neutrino telescopes detect neutrinos indirectly through charged leptons produced in weak interactions. In transparent media, tracks of relativistic particles can be reconstructed by detecting the light produced via the Cherenkov effect, with a three-dimensional array of optical sensors. The type of the interaction, charged current or neutral current, and the neutrino flavour greatly affect the signature of the neutrino event in the detector. Neutral current interactions are similar for all flavours. In this case the neutrino will react with a nucleon, resulting in a lower energy scattered neutrino and a hadronic shower in the final state:

$$
v_{l}\left(\bar{v}_{l}\right)+N \rightarrow v_{l}\left(\bar{v}_{l}\right)+X \quad l=e, \mu, \tau .
$$

Charged current interactions produce a relativistic charged lepton and a hadronic shower:

$$
\nu_{l}\left(\bar{v}_{l}\right)+N \rightarrow l(\bar{l})+X \quad l=e, \mu, \tau .
$$

The emerging lepton inherits the flavour of the incident neutrino. Electron-neutrinos will therefore produce electrons and thereby an electromagnetic shower overlapping with the hadronic shower. Tau neutrinos will produce a $\tau$ lepton which will travel a certain distance and then decay, producing, among other possibilities, another hadronic shower (depending on the type of decay). This type of event with two separated hadronic showers is often called a "double-bang" event. On the other side, the charged current interaction of a muon neutrino results in a muon that at an energy around $1 \mathrm{TeV}$ has path lengths in water of the order of a few kilometers, providing a long lever arm for direction reconstruction. These track-like events are considered the golden channel for neutrino astronomy (see Sect. 3).

High energy neutrino astronomy requires detector volumes of the $\mathrm{km}^{3}$ scale hosted in deep water or in deep

This is an Open Access article distributed under the terms of the Creative Commons Attribution License 4.0, which permits unrestricted use, distribution, and reproduction in any medium, provided the original work is properly cited. 


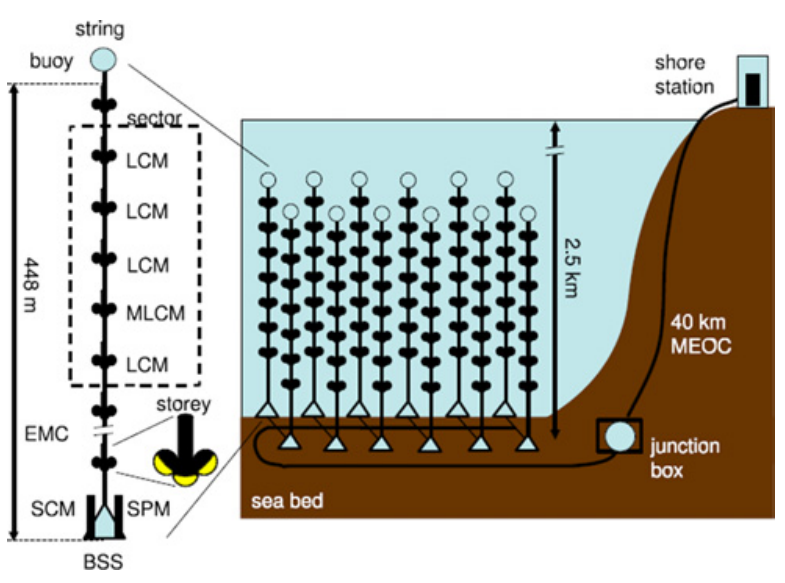

Figure 1. A schematic view of the ANTARES detector layout. The main elements of the ANTARES detector are outlined in the figure.

Antarctic ice, where several thousands of metres of water (or ice) reduce the flux of atmospheric muons by several orders of magnitude. Since neutrinos are the only particles that can pass through the whole Earth, neutrino telescopes look mainly at the up-going neutrinos coming from the opposite hemisphere. An unavoidable background in astrophysical neutrino searches is due to neutrinos produced by the interaction of cosmic ray with the atmosphere. However, the energy spectrum of atmospheric neutrinos is softer than the expected spectrum of cosmic neutrinos. This is key for a possible discrimination. A search bin around the source position can be also used.

\section{ANTARES detector}

The ANTARES detector [2], located $40 \mathrm{~km}$ off the French town of Toulon at a depth of $2475 \mathrm{~m}$, is the first operational Neutrino Telescope in the Mediterranean Sea. It was completed in May 2008 with a total of 885 Optical Modules (OMs) looking $45^{\circ}$ downward and distributed along 12 vertical detection lines. Figure 1 shows a schematic view of the detector.

An OM consists of a 10-inch photomultiplier housed in a glass sphere together with its base, a special gel for optical coupling and a $\mu$-metal cage for magnetic shielding. The OMs are grouped in 25 triplets (or storeys) on each line, except for one of the lines which contains only 20 optical storeys because acoustic devices are installed. Each line has a length of $450 \mathrm{~m}$ and is kept taut by a buoy located at its top. The lower $100 \mathrm{~m}$ are not instrumented. The distance between triplets is $14.5 \mathrm{~m}$ and the separation between the lines ranges from 60 to $75 \mathrm{~m}$, reaching an instrumented volume of about 0.025 cubic kilometers. The lines are connected to a central junction box, which in turn is connected to shore via an electrooptical cable.

\section{Point source analysis}

Point source searches look for a statistical excess originating in narrow sky regions where the sources are located. These searches can be performed either blindly over the full sky or in the direction of a priori candidate source locations e.g. corresponding to known gamma-ray emitters.

The results of the search for cosmic point-like neutrino sources using six years of data collected by the ANTARES neutrino telescope are described in [3]. The high quality runs selected in the measurement period from 2007 to 2012 correspond to a total livetime of 1338 days. Only muon neutrinos are considered in this analysis since they offer a better angular resolution. These track-like events are reconstructed using the combined informations of the photomultiplier spatial positions and the Cherenkov photon arrival times. A detailed description of the multistep reconstruction algorithm can be found in [4]. For each fitted track the algorithm provides a quality parameter $\Lambda$ and an estimate of the uncertainty on the reconstructed track direction $\beta$.

Neutrino candidates are selected following a blind procedure before performing the data analysis. To select the events cuts are applied on $\Lambda, \beta$ and on the reconstructed zenith angle $\theta$ in order to optimize the discovery potential, i.e. the neutrino flux needed to make a $5 \sigma$ discovery in $50 \%$ of the experiments. The resulting cuts $\left(\Lambda>-5.2, \beta<1^{\circ}\right.$ and $\left.\cos \theta<0.1\right)$ lead to a final sample of 5516 events having a median angular resolution of $0.38^{\circ}$ (for an assumed $E^{-2}$ source spectrum).

The search algorithm is based on an unbinned maximum likelihood which includes the information on the point-spread function and on the expected background rate as a function of the declination and of the number hits used in the track reconstruction, that is a proxy for the energy of the event. Two different searches are performed:

Full-sky search. looking for en excess of events over the expected background of atmospheric neutrinos and muons in the whole ANTARES visible sky. The most significant cluster is found at $(\alpha, \delta)=\left(-46.8^{\circ},-64.9^{\circ}\right)$ with a posttrial p-value of $2.7 \%$ (significance of $2.2 \sigma$ using the twosided convention). The upper limits at the $90 \%$ confidence level (C.L.) on the muon neutrino flux from point sources evaluated for declination bands of $1^{\circ}$ are shown in Fig. 2 as light-blue markers.

Candidate-list search. looking for events in the directions of a predefined list of 50 candidate sources of interest which are known gamma-ray emitters and potential sites for hadronic acceleration. No significant excess was found in any of these 50 directions, and upper limits have consequently been derived for these candidate sources (blue squares in Fig. 2).

\section{Search for diffuse neutrino fluxes}

Diffuse flux analyses look in the all sky integrated flux for an excess of events that would reflect the existence of a diffuse neutrino flux from the bulk of unresolved astrophysical sources. This excess would become apparent above a certain energy threshold, depending on the absolute normalization and spectral index of the cosmic neutrino flux. 


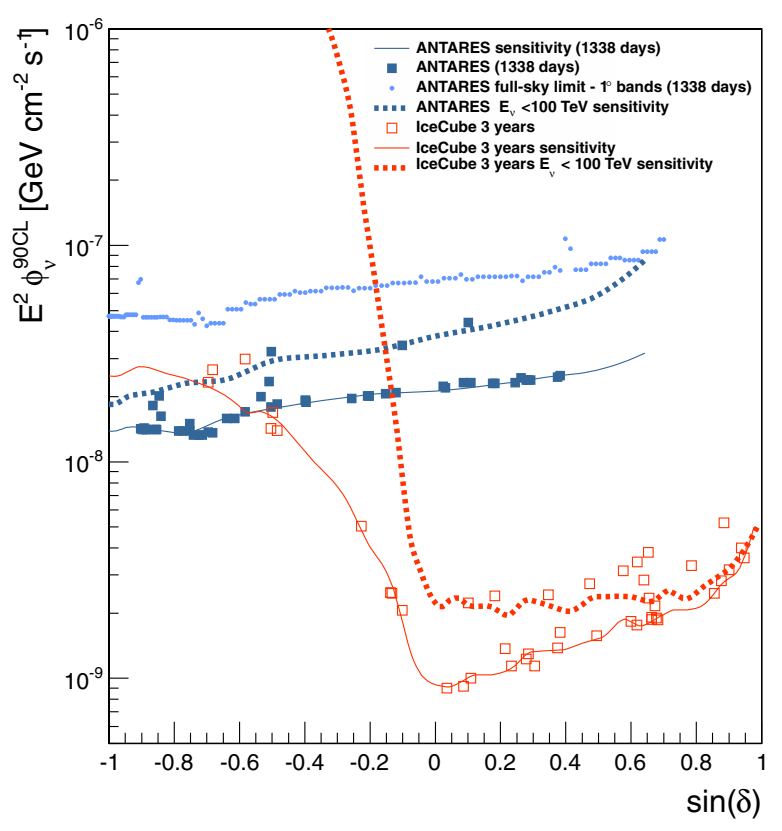

Figure 2. $90 \%$ C.L. flux upper limits and sensitivities on the muon neutrino flux for six years of ANTARES data. IceCube results are also shown for comparison. The light-blue markers show the upper limit for any point source located in the ANTARES visible sky in declination bands of $1^{\circ}$. The solid blue (red) line indicates the ANTARES (IceCube) sensitivity for a point-source with an $E^{-2}$ spectrum as a function of the declination. The blue (red) squares represent the upper limits for the ANTARES (IceCube) candidate sources. Finally, the dashed dark blue (red) line indicates the ANTARES (IceCube) sensitivity for a point-source and for neutrino energies lower than $100 \mathrm{TeV}$, which shows that the IceCube sensitivity for sources in the Southern hemisphere is mostly due to events of higher energy. The IceCube results were derived from [5].

In the following subsections the diffuse neutrino flux search conducted by analyzing separately shower-like and track-like events are described, but studies towards a combined track-shower diffuse neutrino search are ongoing. Moreover, the search for neutrino flux from two extended region of the sky (the Fermi Bubbles and the Galactic plane areas) are also discussed. All the analyses adopt a blind strategy in which the cut is optimized using simulated events only and then applied to the data.

\subsection{Muon neutrino diffuse flux analysis}

The first ANTARES limit obtained with the 2008-2009 data [6] has been updated adding two more years of data reaching an equivalent livetime of 885 days [7]. The two analyses use the same statical method based on the model rejection factor procedure [8] but the recent analysis uses a new energy estimator. Here the muon energy deposit per unit path length is approximated by an estimator which can be derived from measurable quantities such as the amplitude of the hit PMTs [9]. The energy estimator is used to determine the cut yielding the best sensitivity assuming an $E_{v}^{-2}$ source spectrum. In the considered live time and with the track quality cuts applied, the conventional atmospheric neutrinos (simulated according

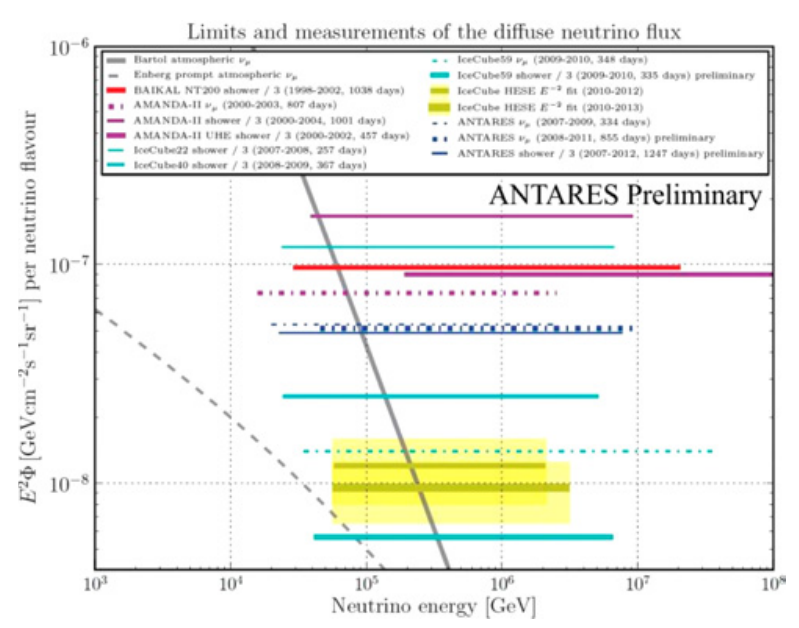

Figure 3. ANTARES 90\% C.L. flux upper limits for an $E^{-2}$ spectrum (blue lines), compared to upper limits set from other experiments. Limits from all flavor shower analyses (solid lines) are divided by 3 (i.e. they are given per flavor) to be able to compare them to limits from muon neutrino analyses. Only the IceCube HESE analyses (yellow lines) are not upper limit but fits of flux expectation [1,11]. All other lines mark upper limits sorted by experiments BAIKAL [12], AMANDA [13-15], IceCube [16,17] and ANTARES [6,7]. For comparison reason the atmospheric fluxes (grey lines) as calculated by Bartol [10] and Enberg [20] have been added. Plot taken from [21].

to the Bartol flux [10]) revealed a $28 \%$ deficit, well within uncertainties, with respect to the data. Therefore the predicted background from the simulation is normalised to the data. After normalization, 8.4 atmospheric events were expected and 8 events were observed after unblinding in the high energy region. This translates into a 90\% C.L. upper limit of

$$
E_{v}^{2} \frac{d N}{d E_{v}}=5.1 \times 10^{-8} \mathrm{GeV} \mathrm{cm}^{-2} \mathrm{~s}^{-1} \mathrm{sr}^{-1}
$$

in the energy range $45 \mathrm{TeV}-10 \mathrm{PeV}$, as indicated in Fig. 3. This upper limit takes into account the systematic uncertainties on the background and signal estimations.

\subsection{All flavours diffuse flux analysis with showers}

The shower reconstruction method used in the current analysis [21] is a two-step maximum likelihood fit that makes use of Probability Distribution Function (PDF) tables extracted from the simulations. After a hit selection that attempts to identify shower pulses among all pulses, a vertex and interaction time reconstruction is performed. The PDF table used in this step stores the probabilities that a single hit has been created from a certain shower vertex and interaction time assumption. Then, the neutrino direction and shower energy are reconstructed using the previously fitted vertex as fixed input. Here the table relates the shower energy to the emission angle of the photons and to the total charge at the vertex that would have been necessary to detect this particular pulse. In the region of interest, with shower energy between $1 \mathrm{TeV}$ and $1 \mathrm{PeV}$, the shower energy reconstruction algorithm achieves 
0.2-0.3 resolution in $\log _{10} E_{\text {shower }}$ with a slight underestimation. The reconstruction of the neutrino direction yields $6^{\circ}$ median resolution for showers with energy below $100 \mathrm{TeV}$, but worsens for higher energies. At $1 \mathrm{PeV}$ the median resolution is approximately $25^{\circ}$. The given values refer to events that have passed an upper cut on the final reduced log-likelihood value of the vertex fit. With this cut applied about $10 \%$ (at $1 \mathrm{TeV}$ ) to $60 \%$ (at $1 \mathrm{PeV}$ ) of all detected shower events survive.

The analysis was applied to 2007-2012 data with a total livetime of 1247 days. Shower events preselection was done using a cut on vertex log-likelihood and requiring that signal hits were present at least on three different detector lines. This reduces significantly the track events contribution including atmospheric muon background. Additionally, it was required that the reconstructed vertex position was not closer than $15 \mathrm{~m}$ to any of the PMTs to exclude events produced due to the sparks in optical modules.

MRF optimisation is done on the fitted shower energy and the zenith angle of the neutrino direction. The cuts that minimize the MRF are $\theta>94^{\circ}$ end $E_{\text {shower }}>10 \mathrm{TeV}$. After the unblinding 8 events were observed where $5 \pm$ 3 events are expected from the atmospheric background (muons, conventional neutrinos as predicted by the Bartol group [10] and prompt neutrinos as predicted by Enberg et al. [20]). The excess over background has a significance of $1.5 \sigma$. For an unbroken $E^{-2}$ spectrum the obtained $90 \%$ C.L. limit on the diffuse cosmic neutrino flux per flavour is

$$
E_{v}^{2} \frac{d N}{d E_{v}}=4.9 \times 10^{-8} \mathrm{GeV} \mathrm{cm}^{-2} \mathrm{~s}^{-1} \mathrm{sr}^{-1}
$$

in the energy range from $23 \mathrm{TeV}$ to $7.8 \mathrm{PeV}$. Also in this case the systematic errors for signal and background are taken into account.

\subsection{Fermi bubbles}

The Fermi-LAT data has revealed a large $(\sim 10 \mathrm{kpc})$ bilateral structure originating from the Galactic Centre and perpendicular to the Galactic plane (and therefore, visible mostly in the southern hemisphere). This structure is generally referred to as Fermi Bubbles (FBs) and its origin is still debated. Several proposed scenarios invoke hadronic mechanisms in which accelerated cosmic rays interact with the interstellar medium, producing pions which would in turn generate neutrinos along the observed gamma-rays [22-24]. A dedicated search has been performed with the 2008-2011 data sample of ANTARES, taking advantage of the good sensitivity of the detector to the central region of our Galaxy; the details of the analysis can be found in [25]. The analysis is applied to upgoing track-like events. The background is estimated from the events observed in areas called offzones with the same size and shape as the FBs regions (on-zone). The off-zones are defined using the same solid angle in local coordinates as the on-zone events, but shifted with some fixed delay in time. This ensures that the offzones and the on-zones have the same expected number of background events since it depends on the efficiency of the detector, which is a function of the local coordinates only.

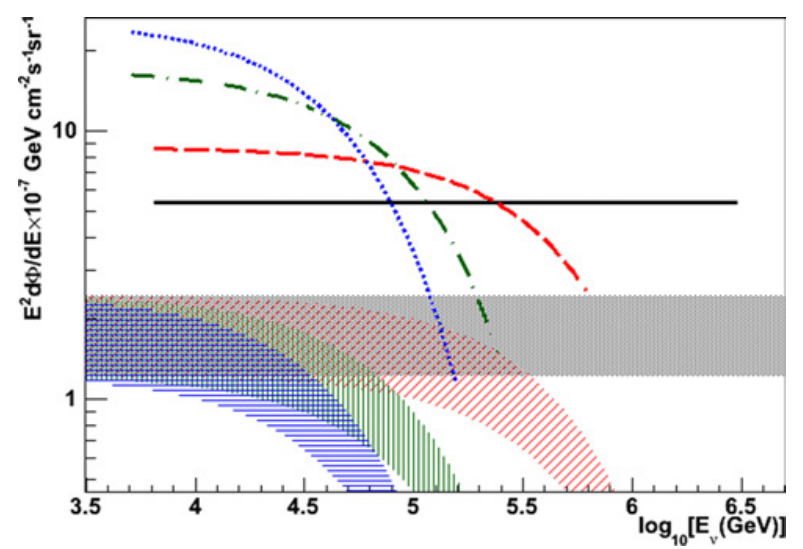

Figure 4. Upper limits on the neutrino flux from the Fermi Bubbles for different cutoffs: no cutoff (black solid), $500 \mathrm{TeV}$ (red dashed), $100 \mathrm{TeV}$ (green dot-dashed), $50 \mathrm{TeV}$ (blue dotted) together with the theoretical predictions for the case of a purely hadronic model (the same colours, areas filled with dots, inclined lines, vertical lines and horizontal lines respectively). The limits are drawn for the energy range where $90 \%$ of the signal is expected.

No statistically significant excess of events above the chosen energy cut was observed in the on-zone with respect to the average expected number from the offzones. The corresponding upper limits on the neutrino flux from the FBs have therefore been derived for different assumptions on the energy cutoff at the source, as shown in Fig. 4. Some of these limits are relatively close to the expected fluxes, suggesting that at least part of the phase space for hadronic models of gamma-ray emission in the FBs could be probed with the full ANTARES data set, or after about 1 year of operation of the next-generation KM3NeT neutrino telescope [26].

\subsection{Galactic plane analysis}

A guaranteed neutrino flux from the Galactic plane is expected thanks to cosmic rays interacting with the matter of the Milky Way. The 2007-2012 ANTARES data were analyzed to look for a diffuse Galactic Plane (GP) neutrino flux [27]. Three theoretical models are considered [28-30]. In the last model, the assumed magnetic field configuration gives rise to the drift of high energy cosmic rays to the Galactic Centre (Drift model). The cosmic ray fluxes obtained from these models are converted to a neutrino flux and for comparison also the gamma-ray data from Fermi-LAT [31] are used for the neutrino flux estimation assuming a pion origin for both gamma-rays and neutrinos. The predicted neutrino fluxes are shown in Fig. 5.

As in the FBs analysis the background is estimated from different off-zones equivalent to the on-zone. The signal region size is optimized considered only for the Drift model as separate optimizations for each model have not shown any significant sensitivity improvements. The best MRF was obtained for the region centred around the Galactic Centre with a size of $78^{\circ}$ in longitude and $9^{\circ}$ in latitude. This region size allows 8 off-zones to be used for background estimation. The expected sensitivity for an $E^{-2.7}$ flux with arbitrary normalization after the final 


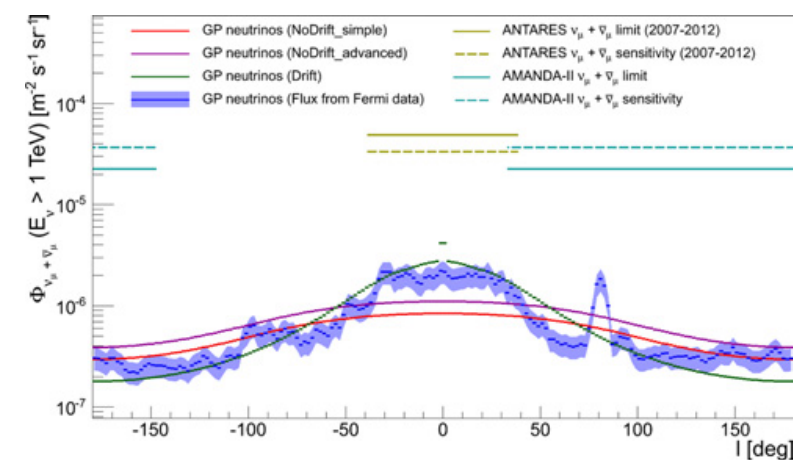

Figure 5. Sensitivity and upper limits (for an $E^{-2.7}$ flux with arbitrary normalisation) for ANTARES versus Galactic longitude together with the neutrino fluxes extracted from different galactic plane models [28-30] and from the Fermi-LAT [31] data, averaged over Galactic latitude and integrated above $1 \mathrm{TeV}$; also shown is the sensitivity and upper limits set by the AMANDA-II experiment [32].

optimization is presented in Fig. 5. After the unblinding 177 events have been measured in the signal region and 166 on average in 8 off-zones giving a significance of $0.8 \sigma$. Final upper limits with $90 \%$ C.L. are shown in Fig. 5.

\section{Multimessenger neutrino searches}

Neutrino telescopes also develop specific strategies to look for neutrinos with timing and/or directional correlations with known (or potential) sources of other cosmic messengers. The limited space-time search window allows a drastic reduction of the atmospheric background, therefore enhancing the sensitivity to faint signals that would have remained undetected otherwise. This method has been used in ANTARES e.g. to put upper limits on the neutrino flux from known gamma-ray transient or flaring sources (see Sects. 5.1 and 5.2).

Alternatively, the occurrence of a special event in a neutrino telescope (such as the near-simultaneous arrival of two or more neutrinos from the same direction) could indicate that a highly energetic burst has occurred and may be used as a trigger for optical, X-ray, and gamma-ray follow-ups (see Sect. 5.3).

Coincident searches of neutrinos and gravitational waves are yet another example of a multi-messenger strategy that could help reveal hidden sources, so far undetected with conventional astronomy (see Sect. 5.4).

\subsection{Neutrinos from GRBs}

A first search for muon neutrinos in correlation with a sample of 40 GRBs that occurred in 2007, while the detector was still in a reduced, 5-line configuration, is described in [33]. A second, more sophisticated search has been performed recently on the 2008-2011 data sample, using a set of 296 long GRBs representing a total equivalent live time of 6.6 hours [34]. In contrast to the previous analyses, this search has for the first time been optimised for a fully numerical neutrino-emission model dubbed as NeuCosmA, which includes Monte Carlo simulations of the full underlying photohadronic interaction processes [35]. The optimisation relied on an Extended Maximum Likelihood Ratio built with an $a$ priori knowledge of the background as estimated using data off-window.

No coincident neutrino event is found within $10^{\circ}$ of any of the GRBs in the sample, and 90\% C.L. upper limits are placed on the total expected flux according to the NeuCosmA model as well as to the commonly used model from [36]. Although not competitive with the IceCube limits [37], the ANTARES results concern a different sample of GRBs (only $10 \%$ of them being also in IceCube's field of view).

\subsection{Neutrinos from flaring sources}

Specific searches have been performed during periods of intense activity of some sources, as reported by X-ray and/or gamma-ray observatories:

- A search for neutrinos in coincidence with 10 flaring blazars chosen on the basis of their FermiLAT light curve profiles was performed using data from 2008. By searching for neutrinos during the high state periods of the AGN light curve, the sensitivity to these sources was improved by about a factor of two with respect to a standard timeintegrated point source search. For one of these AGNs (3C279), one neutrino event was found to be in spatial $\left(0.56^{\circ}\right)$ and time coincidence with a flare. The post-trial p-value is $10 \%$, and the upper limits derived on the neutrino fluence for these AGNs are presented in [38]. A similar analysis is ongoing on the 2008-2012 period using data from Fermi and from the Cherenkov Telescopes HESS, MAGIC and VERITAS.

- A search for neutrinos in coincidence with X-ray or gamma-ray outbursts (as reported by the RXTE/ASM, Swift/BAT or Fermi/LAT satellites) of 6 microquasars was performed on the 20072010 data [39]. No neutrino events were detected in space-time coincidence with those flares, and the inferred limits are close to predictions [40] which may be tested by ANTARES in the upcoming years, in particular for the two microquasars GX339-4 and CygX-3.

\subsection{Follow-up of neutrino events}

Thanks to the ability of its data acquisition system to filter events in real-time, ANTARES can also trigger an external detector on the basis of "golden" neutrino events selected by a fast, online reconstruction procedure [41]. These events can be neutrino doublets coincident in time (within 15 minutes) and space (within $3^{\circ}$ ) or single highenergy neutrinos (typically above $5 \mathrm{TeV}$ ). Neutrino events closer than $0.3^{\circ}$ to a local galaxy (within $20 \mathrm{Mpc}$ ) now also generate an alert. The $2 \pi$ sr instantaneous sky coverage and the high duty cycle of ANTARES are important assets for such neutrino-triggered multi-messenger programs.

Since 2009, alerts have been sent on a regular basis $(\approx 25$ per year) to a network of fast-response, wide-field $\left(1.9^{\circ} \times 1.9^{\circ}\right)$ robotic optical telescopes (TAROT, ROTSE and ZADKO) and more recently also to the SWIFT/XRT 
telescope. No optical counterpart has been observed so far in association with any of the ANTARES neutrino alerts, and limits on the magnitude of a possible GRB afterglow have been recently set [42].

\subsection{Joint searches for neutrinos and gravitational waves}

Both gravitational waves (GW) and high-energy neutrinos (HEN) are cosmic messengers that can traverse very dense media and travel unaffected over cosmological distances, carrying information from the inner regions of astrophysical engines. They could also reveal new, hidden sources that have not been observed by conventional astronomy. This has motivated the development of joint searches between ANTARES and the last-generation GW interferometers VIRGO [43] and LIGO [44], which have been operating concurrently in the past few years. A review on the motivations and challenges of joint GW+HEN astronomy can be found in [45].

The first joint GW+HEN search uses concomitant data taken with the three detectors during 2007, during the VIRGO VSR1 and LIGO S5 science runs, while ANTARES was operating in a 5-line configuration. It consists of an event-per-event search for a GW signal correlating in space and time with a given HEN event considered as an external trigger. No coincident GW+HEN event was observed in this 2007 dataset, which allowed for the first time to place upper limits on the density of joint GW+HEN emitters [46]. More stringent limits will soon become available from a second, optimized search using the data of the full ANTARES telescope in 2009-2010 taken in concomittance with the VIRGO VSR2/VSR3 and LIGO S6 science runs, where all the involved interferometers took data with improved sensitivities [47].

\section{Beyond astrophysics}

Beyond its astrophysics reach, a neutrino detector such as ANTARES also offers broad possibilities for particle physics.

\subsection{Neutrino oscillations}

At the lowest end of its energy sensitivity band, ANTARES can be used for neutrino oscillation studies involving the bulk of detected atmospheric neutrinos. A measurement of the neutrino mixing parameters in the atmospheric sector has been performed with the 2007-2010 data sample, for an overall live time of 863 days [48].

The ANTARES measurement is compatible with the world data giving confidence in the understanding of the detector, even close to the detection threshold. This, in addition, paves the way to additional measurements of the neutrino fundamental parameters by neutrino telescopes, such as the mass hierarchy, which has been recently put forward (e.g. $[49,50])$ thanks to the large measured value of the mixing angle $\theta_{13}$. A feasibility study, dubbed ORCA, for such a measurement with a deep-sea detector is presently being performed in the framework of the KM3NeT Collaboration [51].

\subsection{Dark matter searches}

ANTARES can also be used for searches of dark matter (DM) in the popularly advocated form of WIMPS (weakly interacting massive particles). Such particles get gravitationally trapped in massive bodies such as the Earth, the Sun or the Galactic Center, where they can subsequently self-annihilate, producing neutrinos with energy comparable to the WIMP mass. An excess of neutrinos in the $10 \mathrm{GeV}-1 \mathrm{TeV}$ energy range and in the direction of one such body could therefore provide an indirect signature of the existence of dark matter.

A search in the direction of the Sun has been performed using ANTARES data recorded in 2007-2008, for a total live time of 294.6 days [52]. For each WIMP mass and annihilation channel considered, the quality cuts were designed as to minimize the average $90 \%$ C.L. upper limit on the WIMP-induced neutrino flux. No excess above the atmospheric background was found, yielding upper limits on the spin-dependent and spin-indepedent WIMP-proton cross-section. An improvement in sensitivity is expected in the next update of this analysis with the 2007-2012 data set.

As in the case of the Sun, WIMPs can also accumulate in the centre of the Galaxy and annihilate producing neutrinos. The data recorded by ANTARES between 2007 and 2012, corresponding to a livetime of 1321 days, have been used to search for neutrinos coming from the Galactic Centre [53]. After unblinding, the number of events found are in agreement with those expected for the background and thus limits are imposed on the neutrino fluxes for each of the different annihilation channels.

\section{Conclusions}

The ANTARES detector is currently the largest neutrino telescope that has ever operated in the Northern Hemisphere, and the first to be deployed in the deep sea. Since the connection of its first detection lines in 2007, it has been continuously monitoring the Southern Sky with unprecedented sensitivity, in particular to the $\mathrm{TeV}$ sources lying in the central region of our Galaxy. An extended multi-messenger program complements and expands its astrophysics reach. ANTARES also paves the way to a multi-km ${ }^{3}$ neutrino telescope in the Northern Hemisphere, $\mathrm{KM} 3 \mathrm{NeT}$, to complement the IceCube detector deployed at the South Pole.

Beyond astrophysics, other searches are performed and in particular ANTARES has been able to set very competitive limits on the flux of neutrinos coming from WIMP self-annihilation in the Sun and the Galactic Centre.

More competitive results are expected in the future as ANTARES will continue taking data at least until the end of 2016, when it gets eventually superseded by the nextgeneration $\mathrm{KM} 3 \mathrm{NeT}$ detector.

\section{References}

[1] M.G. Aarsen et al., PRL 113, 101101 (2014)

[2] M. Ageron et al., NIM A 656, 11-38 (2011)

[3] S. Adrían-Martínez et al., The Astrophys. J. Lett. 786, L5 (2014) 
[4] A. Heijboer, PhD thesis, U. van Amsterdam 2004, http://antares.in2p3.fr/Publications/ index.html

[5] M.G. Aartsen et al., Astrophys. J. 779, 132 (2013)

[6] J.A. Aguilar et al., Phys. Lett. B 696, 16 (2011)

[7] V. Kulikoskiy for the ANTARES Collaboration, proceedings of VHEPU2014, to be published

[8] G.C. Hill and K. Rawlings, Astropart. Phys. 19, 393 (2003)

[9] J. Schnabel, NIM A 725, 106-109 (2013)

[10] V. Agrawal et al., Phys. Rev. D 53, 1314 (1996)

[11] M.G. Aarsen et al., Science 342, 1242856 (2013)

[12] Zh.-A. Dzhilkibaev, proceedings of 31st ICRC, Lodz, Poland, July 2009 (arXiv : 0909.5562)

[13] A. Achterberg et al., Phys. Rev. D 76, 042008 (2007)

[14] R. Abbasi et al., Astropart. phys. 34, 420-430 (2011)

[15] M. Ackermann et al., Astrophys. J. 675, 1014-1024 (2008)

[16] R. Abbasi et al., Phys. Rev. D 84072001 (2011)

[17] M.G. Aartsen et al., Phys. Rev. D 89102001 (2014)

[18] M.G. Aartsen et al., Phys. Rev. D 89062007 (2014)

[19] M.G. Aartsen et al., proceedings of 33rd ICRC, Rio de Janeiro 2013 (arXiv: 1309.7003)

[20] R. Enberg, M.H. Reno and I. Sarcevic, Phys. Rev. D 78043005 (2008)

[21] F. Folger, PhD thesis, FAU Erlangen-Nürnberg, 2014

[22] R.M. Crocker, F. Aharonian, PRL 106, 101102 (2011)

[23] B.C. Lacki, arXiv: 1304.6137

[24] S. Thoudam, arXiv:1304.6972

[25] S. Adrían-Martínez et al., EPJ C 74, 2701 (2014)

[26] S. Adrían-Martínez et al., Astropart. Phys. 42, 7 (2013)

[27] E. Visser, $P h D$ thesis, University of Leiden, to be published

[28] G. Ingelman and M. Thunman, Arxiv preprint hep-ph/9604286 (1996)
[29] J. Candia and E. Roulet, JCAP 09, 005 (2003)

[30] J. Candia, JCAP 11, 002 (2005)

[31] M. Ackermann et al., Astropart. Phys. 750, 3 (2012)

[32] J.L. Kelley et al., Proceedings of the 29th ICRC, 5 127-130 (2005)

[33] S. Adrián-Martinez et al., JCAP 1303 (2013) 006

[34] S. Adrián-Martinez et al., A \& A 559, A 9 (2013)

[35] S. Hummer, P. Baerwald and W. Winter, Phys. Rev. Lett. 108 (2012) 231101

[36] D. Guetta, D. Hooper, J. Alvarez-Muniz, F. Halzen and E. Reuveni, Astropart. Phys. 20 (2004) 429

[37] R. Abbasi et al., Nature 484 (2012) 351

[38] S. Adrián-Martinez et al., Astropart. Phys. 36 (2012) 204-210

[39] S. Adrían-Martínez et al. (The ANTARES Collaboration), J. of High Energy Astroph. 3-4, 9-17 (2014)

[40] C. Distefano, D. Guetta, E. Waxman and A. Levinson, Astrophys. J. 575 (2002) 378

[41] M. Ageron et al., Astropart. Phys. 35 (2012) 530

[42] M. Ageron et al., (for the ANTARES, TAROT and ROTSE Coll.), Procs. 33d ICRC, Rio de Janeiro (Brasil), 2013

[43] F. Acernese et al., Class. Quant. Grav. 25 (2008) 184001

[44] D. Sigg, Class. Quant. Grav. 25 (2008) 114041

[45] S. Ando et al., Rev. of Modern Physics 85, 1401-1420 (2013)

[46] S. Adrián-Martinez et al. [LIGO Scientific and Virgo Collaborations], JCAP 1306 (2013) 008

[47] T. Pradier, Procs. 33d ICRC, Rio de Janeiro (Brasil), 2013

[48] S. Adrián-Martinez et al., Phys. Lett. B 714 (2012) 224

[49] E. Akhmedov et al., JHEP 1302, 082 (2013)

[50] D. Franco et al., JHEP 1304, 008 (2013)

[51] P. Kooijman for KM3NeT, 33rd ICRC, id 164

[52] S. Adrían-Martínez et al., JCAP11, 032 (2013)

[53] J.J. Hernandéz-Rey., Procs. Neutrino 2014 